\title{
Mini-invasive treatment of a congenital dacryocystocele by means of microdebrider marsupialization in a newborn
}

\author{
Domenico di Furia ${ }^{1}$, Lorenzo Pignataro ${ }^{2}$, Michele Gaffuri $^{1}$, Silvia Osnaghi ${ }^{1}$, Ludovica \\ Battilocchi $^{1}$, and Giovanna Cantarella ${ }^{1}$ \\ ${ }^{1}$ Fondazione IRCCS Ca' Granda Ospedale Maggiore Policlinico \\ ${ }^{2}$ Fondazione Ca' Granda Ospedale Maggiore Policlinico di Milano
}

July 20, 2021

\begin{abstract}
Congenital dacryocystocele is a rare clinical condition, more commonly unilateral, secondary to the defective canalization of the nasolacrimal duct. In case of failure of conservative treatment, surgical marsupialization is recommended. We describe the case of a 40-days'old male newborn treated by means of microdebrider marsupialization.
\end{abstract}

TITLE: Mini-invasive treatment of a congenital dacryocystocele by means of microdebrider marsupialization in a newborn

AUTHORS: Domenico di Furia ${ }^{1,2 *}$, MD, Lorenzo Pignataro ${ }^{1,2}$, MD, Michele Gaffuri ${ }^{1,2}$, MD, Silvia Gabriella Osnaghi ${ }^{3}, \mathrm{MD}$, Ludovica Battilocchi ${ }^{1,2}, \mathrm{MD}$, Giovanna Cantarella ${ }^{1,2 *}, \mathrm{MD}$

*These two authors equally contributed to this manuscript.

\section{AFFILIATIONS:}

1. Fondazione IRCCS Ca' Granda Ospedale Maggiore Policlinico, Department of Otolaryngology and Head and Neck Surgery, Milan, Italy

2. Università degli Studi di Milano, Department of Clinical Sciences and Community Health, Milan, Italy

3. Fondazione IRCCS Ca' Granda Ospedale Maggiore Policlinico, Department of Ophthalmology, Milan, Italy

CORRESPONDING AUTHOR: Domenico di Furia, MD, Fondazione IRCCS Ca' Granda Ospedale Maggiore Policlinico, Department of Otolaryngology and Head and Neck Surgery, Milan, Italy, Via Francesco Sforza 35, Milan, 20122, Italy. Tel.: +390255032823; e-mail domenico.difuria@unimi.it

KEYWORDS: Ear, Nose and Throat; Paediatrics and adolescent medicine; Ophthalmology

FUNDING SOURCE: This research did not receive any specific grant from funding agencies in the public, commercial, or not-for-profit sectors.

All procedures performed in studies involving human participants were in accordance with the ethical standards of the institutional and national research committee and with the 1964 Helsinki declaration and its later amendments or comparable ethical standards.

Key clinical message: Congenital dacryocystocele is a rare, usually unilateral, clinical condition, secondary to an incomplete canalization of the nasolacrimal duct, which can be treated by means of minimally-invasive marsupialization with microdebrider. 


\begin{abstract}
Congenital dacryocystocele is a rare clinical condition, more commonly unilateral, secondary to the defective canalization of the nasolacrimal duct. In case of failure of conservative treatment, surgical marsupialization is recommended. We describe the case of a 40-days'old male newborn treated by means of microdebrider marsupialization.
\end{abstract}

\title{
CASE REPORT
}

Dacryocystocele is a congenital condition secondary to a defective canalization of the nasolacrimal duct involving the Rosenmuller and Hasner valves ${ }^{[1]}$, thus leading to the subsequent formation of a blueish cystic mass located at the medial cantus with possible extension to the nasal cavity under the inferior turbinate, causing respiratory obstruction ${ }^{[1]}$ in severe cases. The first approach is usually a conservative treatment, while surgery is required when respiratory symptoms occur ${ }^{[2]}$. We here describe the case of a 40-days' old male newborn with a huge right dacryocystocele (Fig.1, Video 1) extending into the nasal cavity with a cystic mass arising from the inferior meatus, deforming the inferior turbinate and completely occupying the anterior part of the nasal cavity (Fig.2). The intranasal cyst was successfully treated by means of a transnasal endoscopic power-assisted marsupialization with microdebrider (Fig. 3a-b); at the end of surgery, the nasolacrimal duct was cannulated to confirm its patency (Fig. 3c-d). This minimally-invasive and atraumatic surgical option allowed the complete marsupialization of the nasal part of the cyst, with the subsequent resolution of respiratory symptoms and medial cantus swelling (Fig. 4a). Follow-up at six months showed no signs of recurrence (Fig. 4b).

\section{CONFLICT OF INTEREST}

Authors have no conflicts of interest to declare.

\section{AUTHOR CONTRIBUTIONS}

DdF, GC: Clinical management of the patient, study conception, acquisition and analysis of data, manuscript draft;

MG: clinical management of the patient, acquisition and analysis of data, manuscript draft;

LP, SO, LB: critical revision of the manuscript;

All authors: review of the final draft of the manuscript, approval of the manuscript's submission.

\section{CONSENT FOR PUBLICATION}

Written informed consent was obtained from the patient's parents for the publication of this case report and the accompanying images.

\section{REFERENCES}

Cavazza S, Laffi GL, Lodi L, Tassinari G, Dall'Olio D. Congenital dacryocystocele: diagnosis and treatment. Acta Otorhinolaryngol Ital 2008;28:298-301.

Khatib L, Katowitz JA, Katowitz WR. The treatment of neonatal and infantile dacryocystoceles and dacryocystitis with the microdebrider. Journal of AAPOS;2018;22(4):e83-e84.

\section{FIGURES LEGENDS}

Video 1: a 40-days' old male newborn with a huge right dacryocystocele extending into the nasal cavity with a cystic mass completely occupying the anterior part of the nasal cavity. The intranasal cyst was successfully treated by means of a transnasal endoscopic power-assisted marsupialization with microdebrider.

Figure 1: Right congenital dacryocystocele. 
Figure 2: Nasal endoscopy showed a cystic mass (asterisk) arising from the inferior meatus, deforming the inferior turbinate (IT) and in contact with the nasal septum (NS), completely occupying the anterior part of the right nasal cavity.

Figure 3: Transnasal endoscopic power-assisted marsupialization of the nasal cyst (a,b); purulent discharge coming from the nasal cyst (b) during the surgical marsupialization with microdebrider; at the end of surgery, the nasolacrimal duct was cannulated to confirm its patency $(c, d)$.

Figure 4: No residual mass was detectable at the end (a) and after six months from surgery (b).

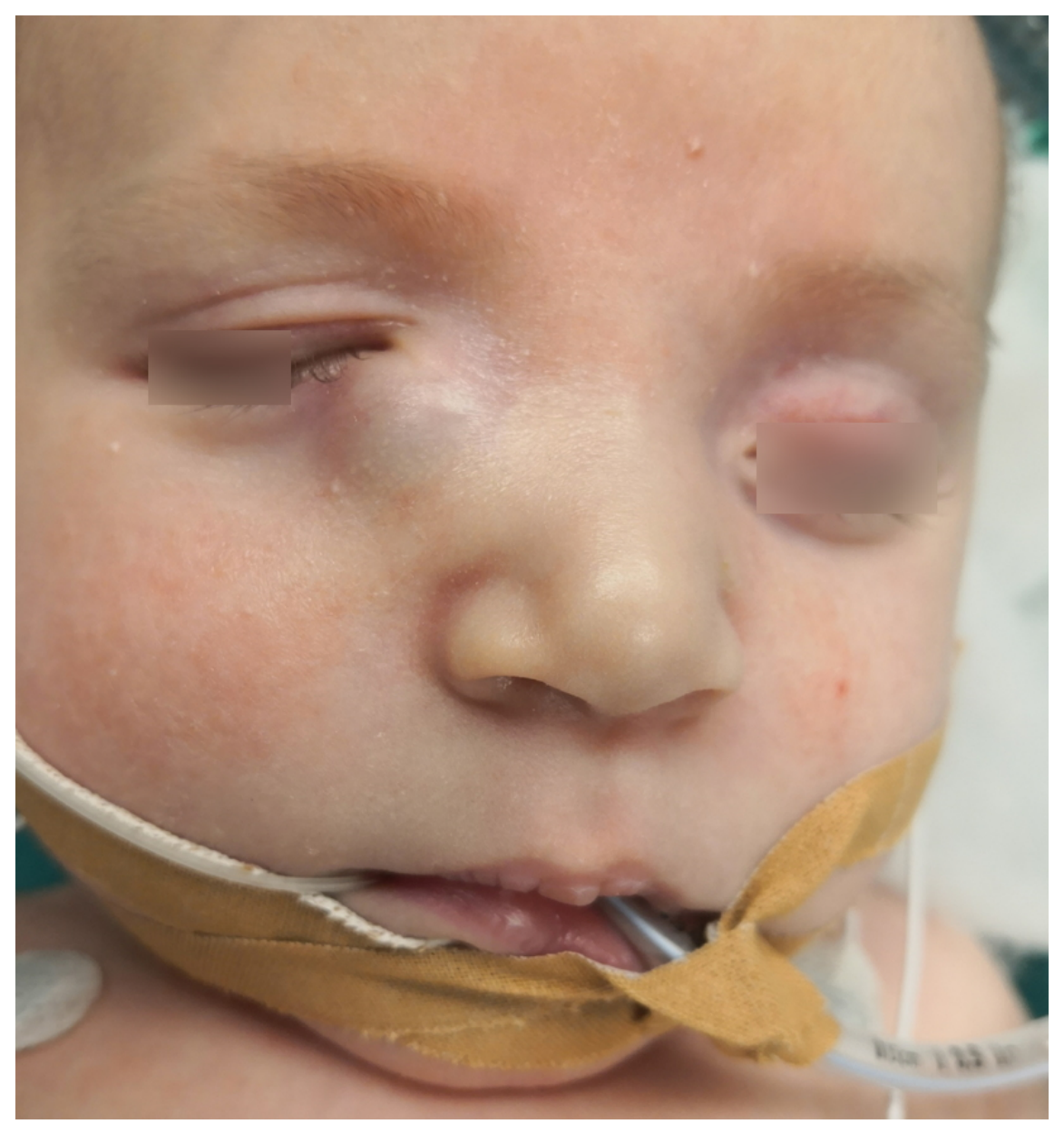




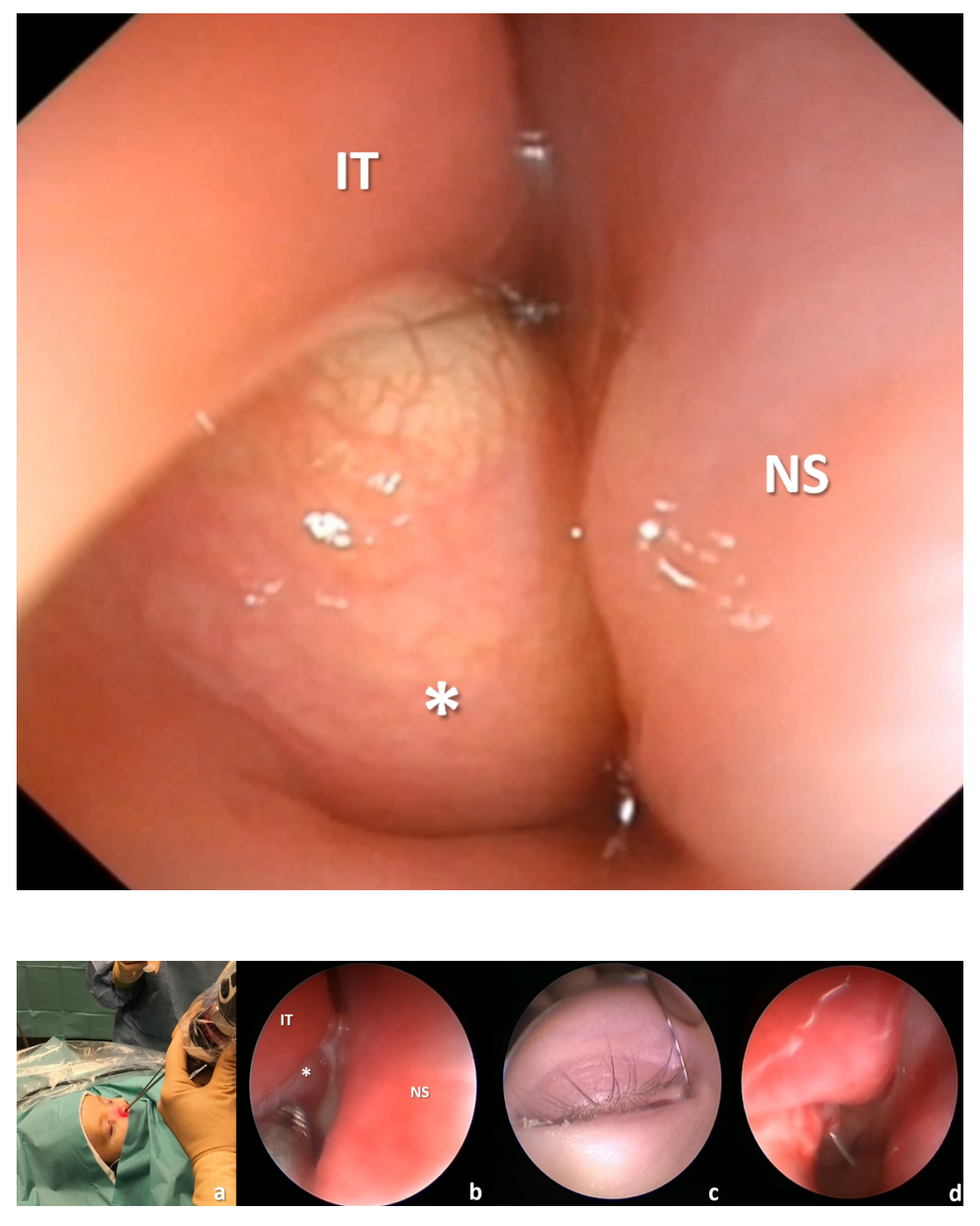




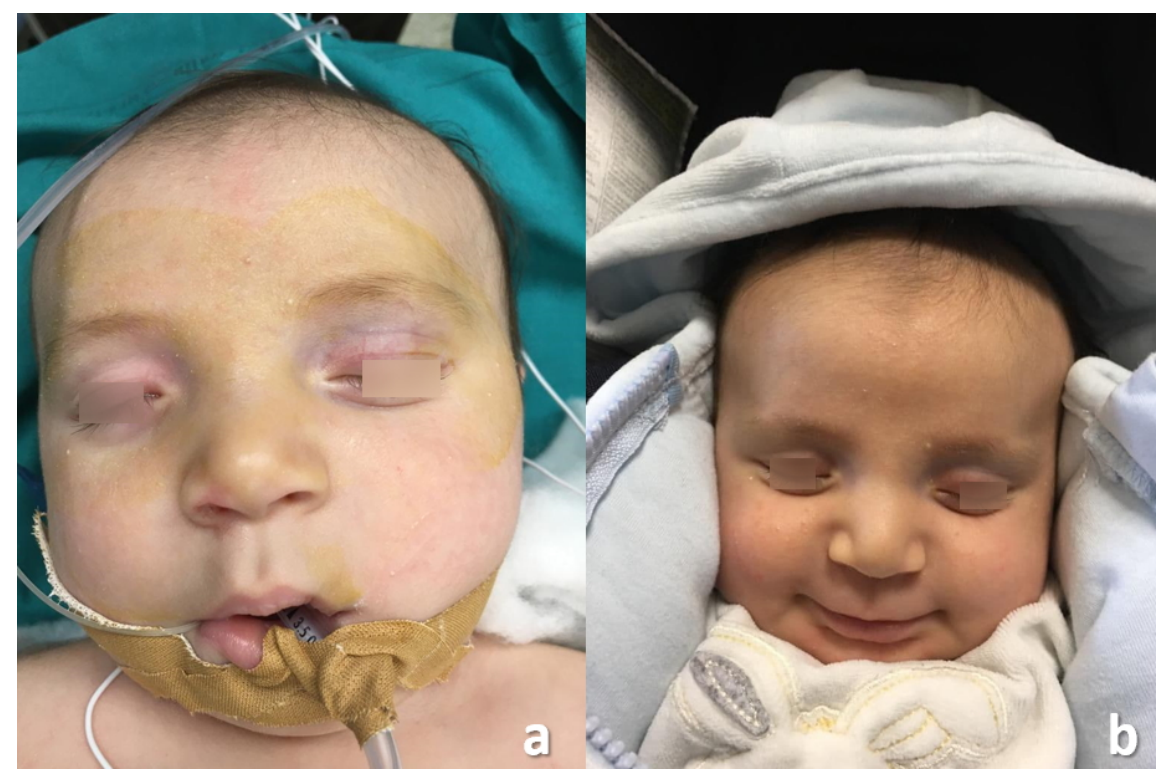

